\title{
Doxorubicin/Mitomycin/Tegafur Regimen
}

National Cancer Institute

\section{Source}

National Cancer Institute. Doxorubicin/Mitomycin/Tegafur Regimen. NCI Thesaurus.

Code C10023.

A chemotherapy regimen consisting of doxorubicin, mitomycin, and tegafur that may be used in the treatment of non-small cell lung cancers (NSCLC). 